\title{
Recurrent Bowen's disease of scalp treated with high dose rate surface mold brachytherapy: a case report and review of the literature
}

\author{
Ajeet Kumar Gandhi, MD!, Macharla Anjaneyulu Laviraj, MSc², Lakhan Kashyap, MBBS', Suvendu Purkait, MD³, \\ Daya Nand Sharma, MD, Addl. Prof.', Prof. Pramod Kumar Julka, MD', Prof. Goura Kishor Rath, MD' \\ 'Department of Radiation Oncology, All India Institute of Medical Sciences, ${ }^{2}$ Medical Physics, Department of Radiation Oncology. \\ All India Institute of Medical Sciences, ${ }^{3}$ Department of Pathology. All India Institute of Medical Sciences, Ansari Nagar, New Delhi, India
}

\begin{abstract}
Our case is a 46-year-old female presenting to us with Bowen's disease of scalp since 5 years. Patient had failed topical therapy with $5 \% 5$-florouracil, $0.1 \%$ tacrolimus and was intolerant to topical imiquimod. At presentation, she had $15 \mathrm{~cm} \times 10 \mathrm{~cm}$ erythematous, hyperpigmented, crusted plaque with irregular border in the superior and lateral aspect of left side of scalp with extension in to forehead. Patient was treated with computed tomography based customized surface mold high dose rate brachytherapy with Iridium-192 to a dose of 35 Gy in 10 fractions (twice daily, 6 hours apart) over 5 days. Patient tolerated the treatment well and showed regression of the lesion with mild dermatitis at the end of treatment. Though dermatitis increased at 2 weeks, at 4 weeks post treatment there was near complete resolution of the lesion with adjacent alopecia. At 8 weeks after completion of the treatment, there was complete resolution of the lesion and patient was asymptomatic. Alopecia in the adjacent area has resolved and the skin pigmentation has begun. Patient is satisfied with both the disease control and the cosmetic outcome of the procedure. Our case report demonstrates successful application of surface mold high dose rate brachytherapy in the treatment of recurrent Bowen's disease of the scalp. Brachytherapy can play an important role in the management of recurrent malignant and premalignant diseases of the complex treatment sites like scalp and it's non-hesitant use should be encouraged in appropriately selected patients at the earliest.
\end{abstract}

Key words: Bowen's disease, scalp, radiation therapy, brachytherapy.

\section{Purpose}

Bowen's disease is synonymous with squamous cell carcinoma in situ and was originally described by James $\mathrm{T}$. Bowen in 1912 [1]. It is a disease of old age (median age of presentation is $6^{\text {th }} / 7^{\text {th }}$ decade of life) with slight female predilection $[2,3]$. The most common sites of affliction are head and neck (29-54\%) and scalp (with a predilection towards male) is involved in around $20 \%$ of the cases [4].

Diagnosis is made clinically based on typical findings of an erythematous, ill-defined, hyperkeratotic plaque like lesion, and additional help of dermoscopy is often sought. A punch biopsy is done, if a confirmation is required in doubtful or recurrent/refractory cases [4]. The risk of progression to invasive squamous cell carcinoma has been reported to be $3-5 \%$ based on retrospective reviews, but it has been reported to be as high as $20 \%$ in some studies $[5,6]$. This mandates the treatment to be instituted as soon as possible after the diagnosis. No standard treatment exists and the treatment of Bowen's disease is widely variable [4]. The usual treatment approach is to use non-invasive topical therapies and reserve other treatments for recurrent/refractory cases. However, treatment options for recurrent/refractory lesion particularly large sized lesions and lesions at difficult sites like lower extremity or scalp are very limited. Surgical excision in these cases not only has poor wound healing rates [7], but also is cosmetically unacceptable. Radiation therapy has been used in these cases with excellent local control and cosmetic outcome [8-10]. Varying radiation treatment techniques has been used, but the reports of computed tomography (CT) based high dose rate (HDR) brachytherapy for the treatment of Bowen's disease of scalp is limited.

We report treatment of a large recurrent Bowen's disease of scalp refractory to topical therapy and not 
amenable to surgical excision with CT based customized HDR (remote after-loaded Iridium-192) surface mold brachytherapy.

\section{Case report}

A 46-year-old female presented with a non-healing, erythematous ulcer on the left side of her scalp for one year to the department of dermatology in our institute. She complained of purulent discharge from the lesion off and on, but there was no history of pain, itching or photosensitivity. No history of trauma to the local site, chronic sun exposure or any chemical exposure could be elicited. There was no history of any co-morbidities or immunosupression (history of recurrent infections, organ transplant etc.). At the time of presentation to dermatology department in 2008, a $1 \mathrm{~cm} \times 1 \mathrm{~cm}$ erythematous lesion with erosion and purulent crust was noted on the antero-lateral aspect of the left scalp. No similar lesion or other lesion was noted in any other muco-cutaneous sites and systemic examination did not reveal any abnormality.

A

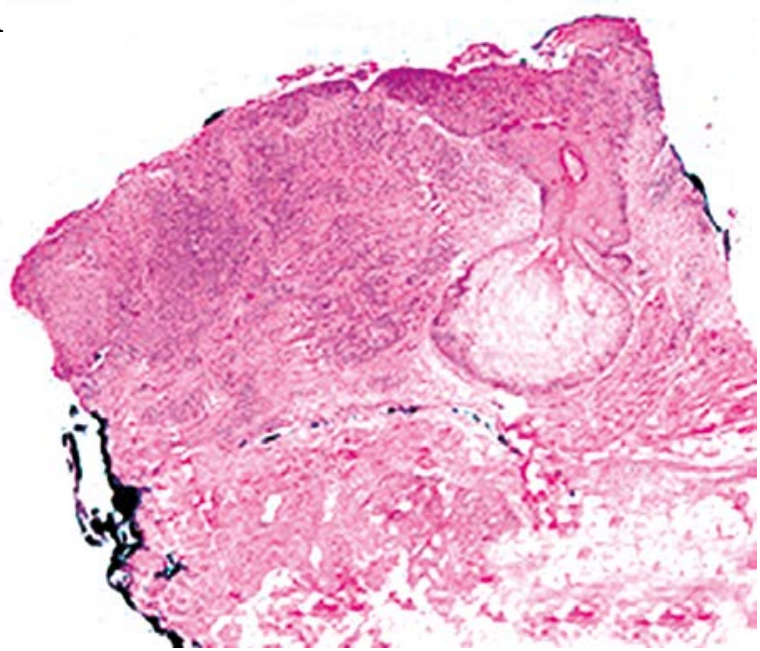

C

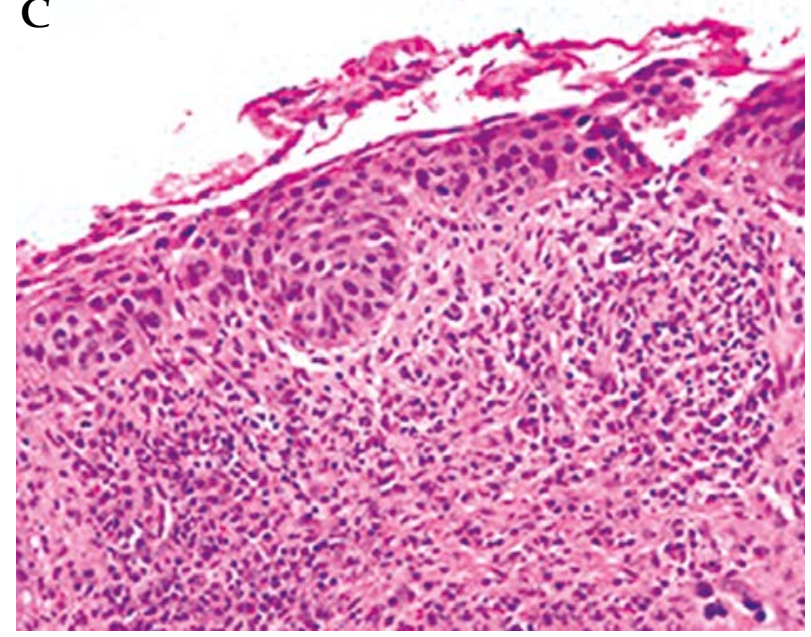

Patient was started on fluticasone (steroid) and mupirocin (antibiotic) and the lesion resolved, but re-appeared after 3 months. At this time, she complained of itching, purulent discharge from the lesion and also burning sensation on sun exposure. Lesion size was $2.5 \mathrm{~cm} \times 2.5 \mathrm{~cm}$ (erythematous with purulent crusting) and a skin biopsy was ordered at this point (June 2009). Skin biopsy (Fig. 1A-D) showed features consistent with diagnosis of Bowen's disease. She received topical 5\% 5-florouracil (in August 2009) and had non-complete resolution of the lesion. Subsequently, the lesion progressed to a size of $5 \mathrm{~cm} \times 5 \mathrm{~cm}$ and $0.1 \%$ topical tacrolimus was tried with minimal response. In view of recurrent nature, a repeat biopsy was done in January 2012, which again revealed same histology. She was started on $5 \%$ imiquimod, to which she developed dermatitis and could not tolerate it well. She also intermittently received steroids, antibiotics, sun protectants etc. throughout this course of treatment. In view of intolerance to therapy/non-responsiveness and progression of the lesion, she was referred to our department in July 2013.
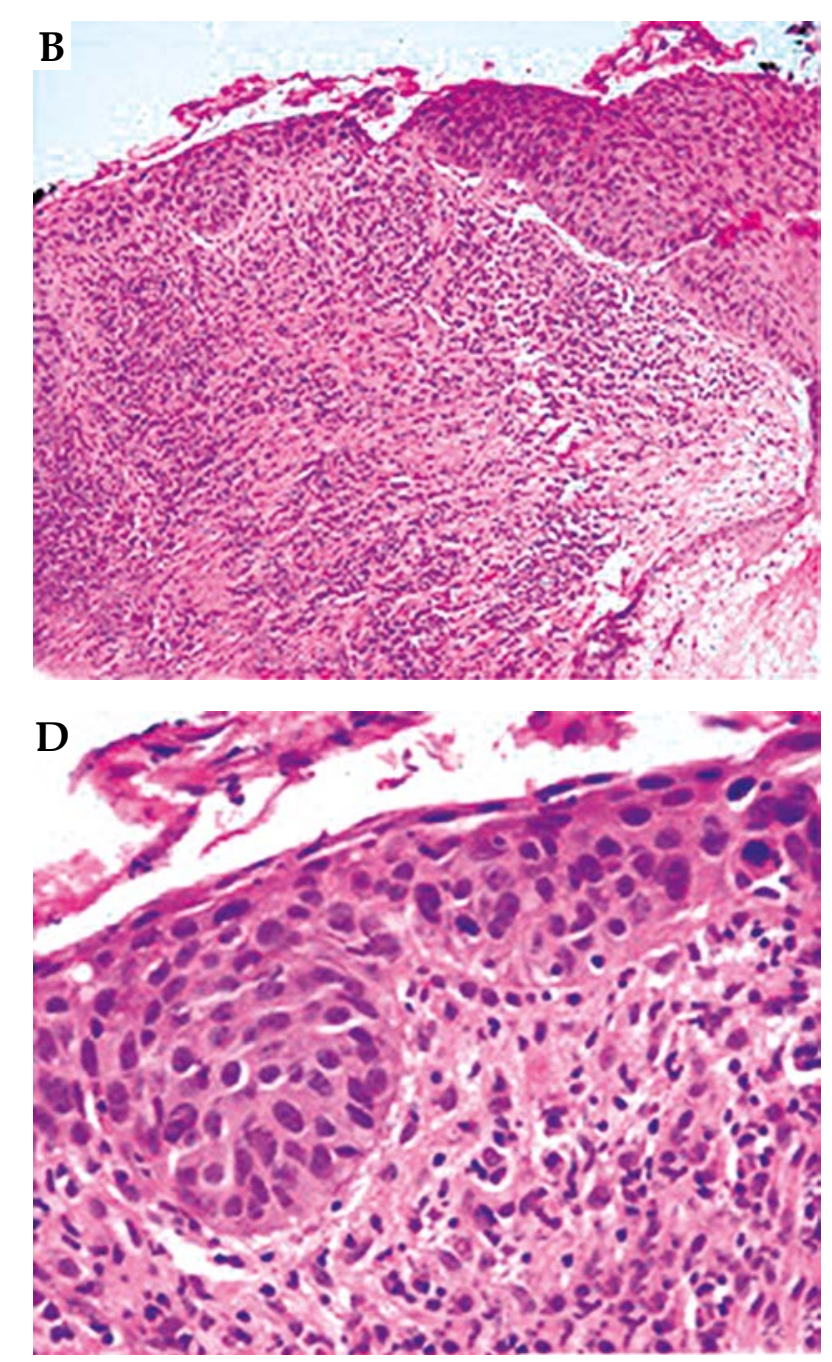

Fig. 1. A) The epidermis show irregular acanthosis with loss of normal maturation pattern $(H \& E \times 4)$. B) Focally the epidermal abnormality also extending into the follicular infandibulam $(\mathrm{H} \& \mathrm{E} \times 10)$. C) Marked cellular disarray in the epidermis with no evidence of invasion $(H \& E \times 20)$. D) The neoplastic cells showing moderate degree of pleomorphism and high nucleo-cytoplasmic ratio. Frequent mitotic figures are seen (arrow) $(H \& E \times 40)$ 
She was evaluated jointly by a radiation oncologist and surgical oncologist. At presentation to us, she had complains of pain, itching, and purulent discharge from the lesion, with exacerbation of symptoms since the last 6 months. On examination, she had a $15 \mathrm{~cm} \times 10 \mathrm{~cm}$ erythematous, hyperpigmented, crusted plaque in the superior and antero-lateral aspect of the left side of scalp with well defined irregular border and extension to forehead (Fig. 2A). No regional lymphadenopathy was noted and rest of the systemic examination was normal. Hematological parameters were normal and HIV (human immunodeficiency virus) was non-reactive. Contrast enhanced CT scan of head revealed no underlying bone erosion. Surgery was deferred in view of large lesion size, cosmetic and reconstructive issues, and patient's un-willingness. A decision was taken to treat the patient with radiotherapy after discussion with the patient and after an informed consent.

A customized thermoplastic immobilization cast of the head was made. Flexible plastic brachytherapy catheters (12 catheters at a distance of $1 \mathrm{~cm}$ from each other) inserted between layers of wax was pasted in close con-
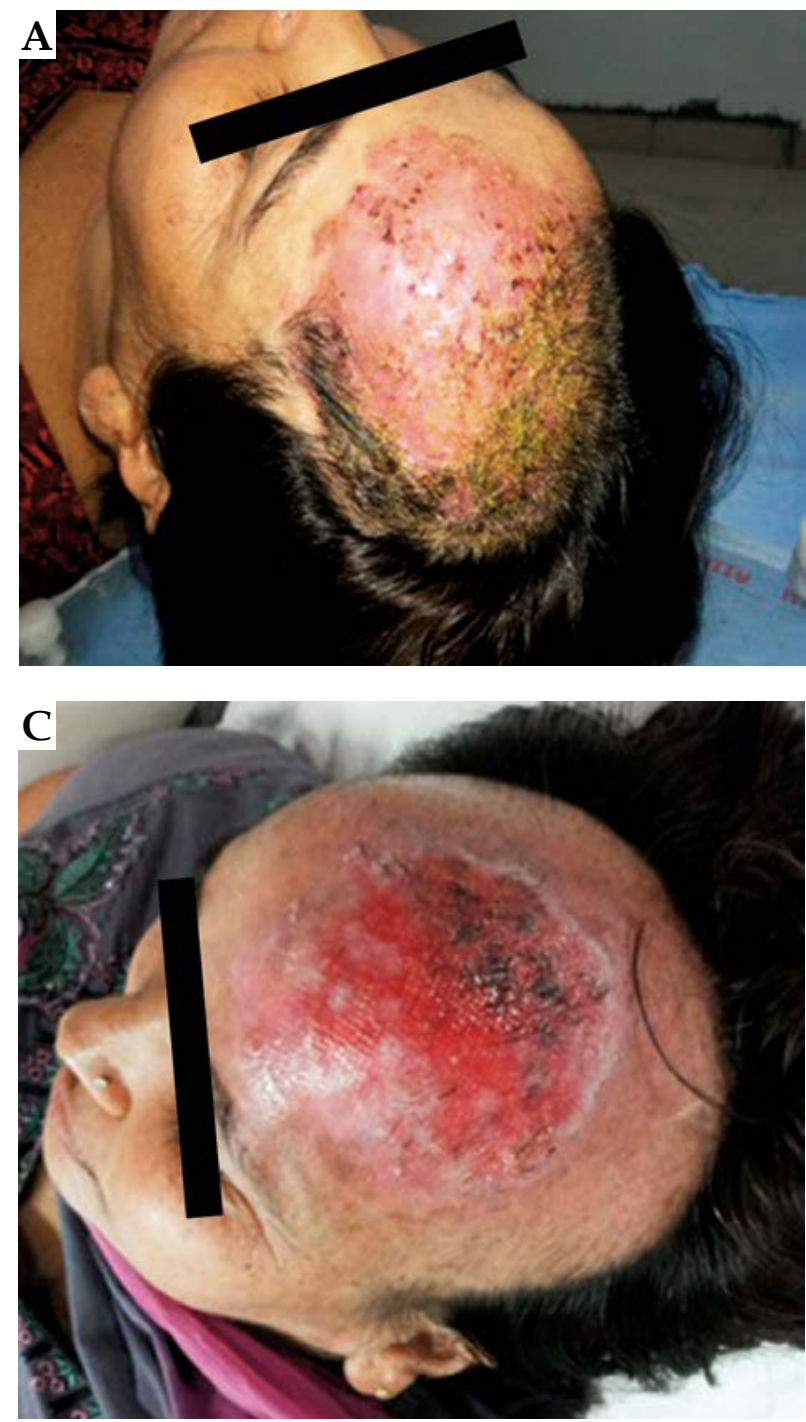

tact with the thermoplastic cast (Fig. 3A). Computed tomography scan (3 $\mathrm{mm}$ thickness) was acquired and a lead wire was also placed around the circumference of the lesion (before the assembled cast was applied) for proper delineation of the treatment area. The planning CT scan was transferred to Oncentra treatment planning system (Nucletron, an Elekta company, Elekta AB, Stockholm, Sweden). Clinical target volume (CTV) was delineated based on the markers and clinical findings. Organs at risk including brain parenchyma, bones of skull, eyes, lens, optic nerves, optic chiasm, and brainstem was also delineated on serial CT slices (Fig. 3B). Catheters were reconstructed with axial and multi-planar views. Dwell positions were created with spacing of $5 \mathrm{~mm}$ and normalization was done using catheter points. Graphical optimization was done to ensure $95 \%$ coverage of the CTV (Fig. 3C). A dose of 35 Gy was prescribed in 10 fractions ( 2 fractions per day, 6 hours apart). Lesion coverage factor [11] was 0.951 ( $\mathrm{TV}_{\mathrm{RI}}$ [volume of target covered by the reference isodose line]/TV [target volume]). Target volume measured $33.59 \mathrm{~cm}^{3}$. Brain parenchyma received a mean
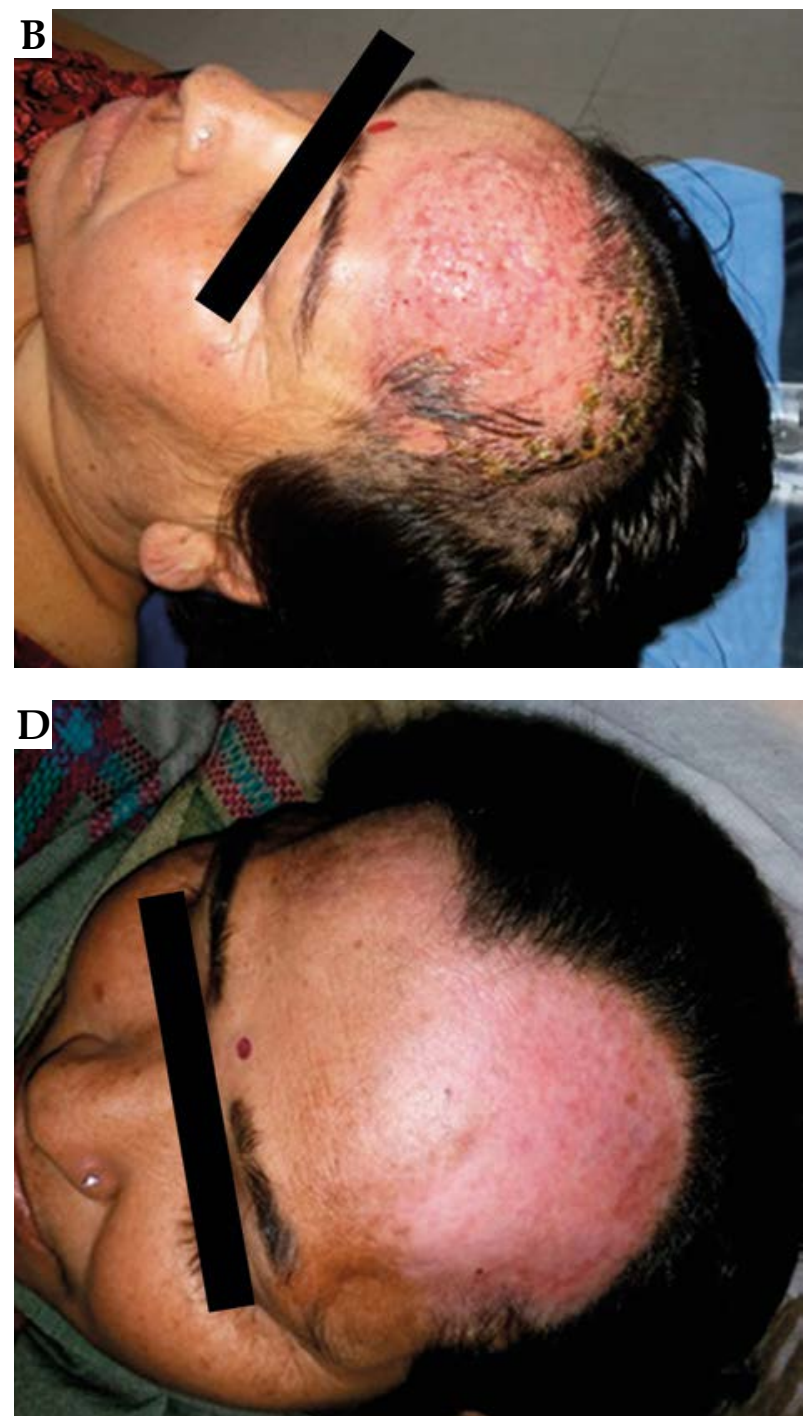

Fig. 2. A) Clinical picture of the patient before radiotherapy treatment. B) On the day of completion of treatment. C) Two weeks after completion of treatment. D) Eight weeks after completion of treatment 

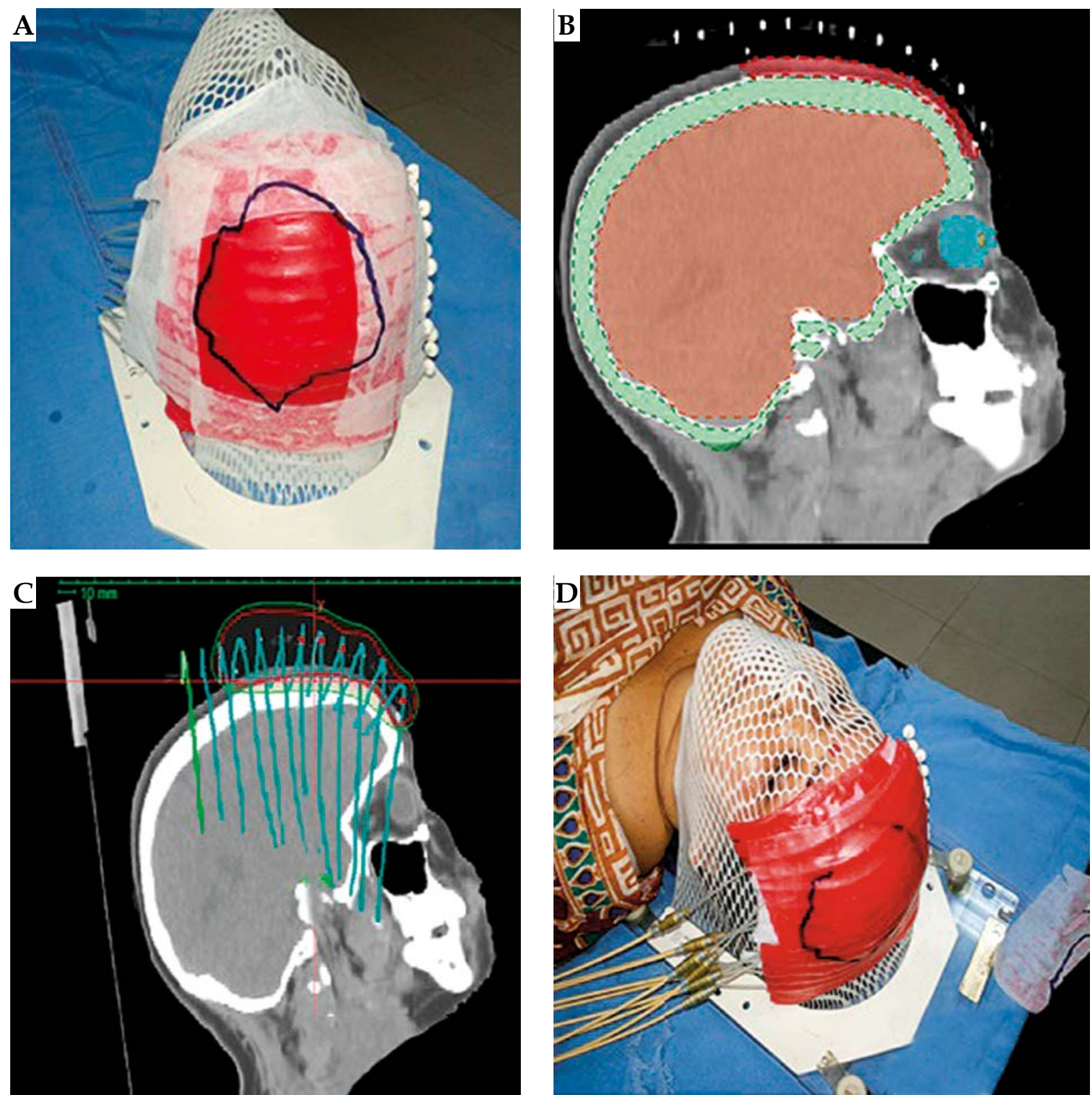

Fig. 3. A) Shows customized mold with plastic brachytherapy catheters in situ. B) Planning CT scan acquired with customized mold. Orange arrow shows the brachytherapy catheters with lead wire and green arrows show the lead wire around the lesion placed to delineate the treatment area. C) Isodose lines (green color shows $80 \%$ and red color shows $100 \%$ isodose lines) for the treatment. D) Shows patient being treated on remote after loading HDR brachytherapy unit

dose of $0.39 \mathrm{~Gy} /$ fraction and dose to whole brain was $0.07 \mathrm{~Gy} /$ fraction of treatment. Rest of the dosimetric data has been summarized in Table 1 . Treatment was administered using an Iridium-192 source (Microselectron HDR, Nucletron, an Elekta company, Elekta AB, Stockholm, Sweden) (Fig. 3D). Treatment time was 10 minutes per fraction including treatment set up time. Patient tolerated the treatment well without any interruption. Figure 2B shows the clinical picture at completion of the treatment. At 2 weeks after completion of the treatment (Fig. 2C), healthy granulation tissue with partial resolution of the lesion was appreciable along with alopecia in the adjacent area. Figure 2D shows complete resolution of the lesion at
8 weeks of treatment. Alopecia was resolved in the adjacent area and there is no evidence of radiation dermatitis or ulceration.

Patient is asymptomatic 14 months post treatment and reports cosmesis as excellent. However, in view of chances of late recurrences and secondary malignancy, she needs to be on long term follow up.

\section{Discussion}

The treatment of Bowen's disease ranges from observation, topical therapies, surgery to radiotherapy [4] based on a number of factors including lesion site, lesion size, 
thickness of lesion, patient's age, and preference, availability of therapeutic modalities, and physician's choice of treatment. Of the local therapies, 5\% Fluorouracil cream $[12,13]$ and $5 \%$ Imiquimod $[14,15]$ usually is sufficient in most of the patients. These topical therapies are not equally tolerated by all patients. Thirty-eight percent of patients in a study by Mackenzie-Wood et al. [16] discontinued 5\% Imiquimod because of side effects of treatment.

Treatment of Bowen's disease of scalp, which is refractory to topical therapies is challenging. Surgical resections, particularly of large lesions are not only cosmetically unacceptable, but are also associated with increased wound complication rates [7]. Chemotherapy or other systemic therapies are usually not indicated for localized premalignant or malignant lesions of scalp. Radiation therapy remains an underutilized treatment option for this disease and is often called in to action when all other treatment options have failed or is not possible.

Radiation therapy for Bowen's disease is not new and is particularly suited for the patients with large, recurrent or multiple tumors at cosmetically sensitive body sites, and more so in patients who refuse surgery or are predisposed to keloids. Radiotherapy has its own advantages and limitations. All the body sites don't tolerate radiation well. Radiation therapy for lower extremity has been associated with poor wound healing. In a study by Dupree et al. [10,17], 25\% of the patients had unhealed lesions after radiotherapy and all of them belonged to lower extremity. Scalp is a difficult site to be treated with radiation therapy owing to its irregular convex contour and its predisposition towards poor wound healing. These limitations are more so in case of lesion involving large areas of scalp.

Limited studies have explored the role of external beam radiotherapy for Bowen's disease of scalp. Twenty percent patients ( 9 patients) in the study by Lukas VanderSpek et al. [9] had lesions of scalp. Patients in this study were treated with orthovoltage $\mathrm{X}$-rays. The median biologically effective dose used in this study was 49.3 Gy (range: 26.4-65.3 Gy). All patients had complete response except one. Radiation therapy has been used in doses ranging from 25-70 Gy with dose per fraction ranging from 2-5 Gy [8]. Dose per fraction more than 4 Gy [9] has been associated with impaired healing and a dose per fraction less than this should always be encouraged. We in our study, used a dose of 35 Gy at 3.5 Gy per fraction and achieved an excellent outcome.

Treatment of superficial skin tumors with mold brachytherapy technique dates back to 1989 by Ashby et al. [18]. These authors treated 642 patients of non-melanoma skin malignancies using wax molds loaded with radon sources. Mold brachytherapy has been tried for sites like oral cavity [19] and mold based surface brachytherapy has been used for scalp irradiation for leukemic infiltrates of the scalp by Liebmann et al. [20]. Gauden et al. [21] reported their experience of 200 patients of non-melanoma skin cancers treated with HDR brachytherapy using a special applicator (Leipzig surface applicator), and reported an excellent local control rate (98\%) and good to excellent cosmesis $(88 \%)$ at a median follow up of 66 months (range 25-121 months). Tormo et al. [22]
Table 1. Doses values to the OARs

\begin{tabular}{lc} 
OARs & Dose $\left(D_{\max }\right)$ \\
\hline Left eye & $36.84 \%$ \\
\hline Left optic nerve & $22.89 \%$ \\
\hline Brainstem & $16.82 \%$ \\
\hline Right eye & $18.89 \%$ \\
\hline Right optic nerve & $19.45 \%$ \\
\hline Skull bone & $90.78 \%$
\end{tabular}

OARs - organ at risk, $D_{\max }$ - maximum point dose received by organ

described results of 32 patients (with 45 non-melanoma skin cancers) treated with Valencia applicator with HDR brachytherapy and reported a $98 \%$ local control rate at a median follow up of 47 months.

Kowalik et al. [23] described CT based HDR surface mold brachytherapy technique for three unfavorably localized malignant lesion and have described in detail the process of manufacturing individual applicator. Similarly, we in our study used CT based plan optimization. Computed tomography based plan helps to more clearly visualize the coverage of intended treatment area and also evaluate irradiation of underlying critical structure.

The time points of evaluation of toxicities and cosmesis in HDR surface mold brachytherapy is also important. Study by Guix et al. [24] illustrates the temporal patterns of treatment complication and cosmesis. Skin erythema and varying degrees of skin ulceration is a rule rather than exception. Skin toxicities should be evaluated at longer time points of 6-12 weeks, as most of the reactions settle and cosmesis is best evaluated. In this study by Guix et al. [24], cosmesis was good or excellent in only $38 \%$ of patients at 2 weeks and was $98 \%$ at 3 months post completion of treatment. Also, no definite guidelines exist to grade reactions in surface mold brachytherapy. However, the definition used by Lukas VanderSpek et al. [9] seems to be reasonable to use. Grade 4 toxicity in this study was defined as necrosis (cartilage/bone damage) and/or ulceration for a duration of $>3$ months. In our patient also, the reaction subsided only by 4 weeks of treatment and complete resolution was seen roughly 6 weeks after the completion of treatment.

Yet another innovative approach to treat areas of face (eyelids, nose, lips etc.) is the application of a specially designed radioactive skin patch coated with high energy beta-emitter holmium-166. Chung et al. [25] used this approach to treat Bowen's disease of face in eight patients (29 sites) with complete resolution in all patients at 1-5 months. The patches were topically applied for 30-60 minutes for a total radiation dose of $35 \mathrm{~Gy}$. All patients developed desquamation, erythema or erosion after 1-2 weeks of treatment, but all at good functional and cosmetic outcomes later on.

In conclusion, based on the available evidence, radiation therapy is a highly effective therapy for Bowen's disease of the scalp. Customized CT based HDR surface mold brachytherapy with Iridium-192 is well tolerated, 
highly reproducible, suited for accurate dose calculation and optimization, is associated with acceptable short term radiation morbidity, and an excellent outcome in terms of local control and cosmesis. It's non-hesitant use should be considered in all patients responding poorly to topical therapies, particularly for cosmetically sensitive areas of body like scalp.

\section{Disclosure}

Authors report no conflict of interest.

\section{References}

1. Bowen JT. Precancerous dermatoses: a study of two cases of chronic atypical epithelial proliferation. J Cutan Dis 1912; 30: 241-255.

2. Eedy DJ, Gavin AT. Thirteen-year retrospective study of Bowen's disease in Northern Ireland. Br J Dermatol 1987; 117: 715-720.

3. Jaeger $\mathrm{AB}$, Gramkow $\mathrm{A}$, Hjalgrim $\mathrm{H}$ et al. Bowen disease and risk of subsequent malignant neoplasms: a population-based cohort study of 1147 patients. Arch Dermatol 1999; 135: 790-793.

4. Morton CA, Birnie AJ, Eedy DJ. British Association of Dermatologists' guidelines for the management of squamous cell carcinoma in situ (Bowen's disease) 2014. Br J Dermatol 2014; 170: 245-260.

5. Kao GF. Carcinoma arising in Bowen's disease. Arch Dermatol 1986; 122: 1124-1126.

6. Peterka ES, Lynch FW, Goltz RW. An association between Bowen's disease and cancer. Arch Dermatol 1961; 84: 623-629.

7. Bell HK, Rhodes LE. Bowen's disease - a retrospective review of clinical management. Clin Exp Dermatol 1999; 24: 338-339.

8. Anna Z, John K, Maria T et al. The potential role of radiation therapy in Bowen's disease: a review of the current literature. Rev Recent Clin Trials 2012; 7: 42-46.

9. Lukas VanderSpek LA, Pond GR, Wells W et al. Radiation therapy for Bowen's disease of the skin. Int I Radiat Oncol Biol Phys 2005; 63: 505-510.

10. Dupree MT, Kiteley RA, Weismantle K et al. Radiation therapy for Bowen's disease: lessons for lesions of the lower extremity. J Am Acad Dermatol 2001; 45: 401-404.

11. Wojcicka JB, Lasher DE, McAfee SS et al. Dosimetric comparison of three different treatment techniques in extensive scalp lesion irradiation. Radiother Oncol 2009; 91: 255-260.

12. Morton C, Horn M, Leman J et al. Comparison of topical methyl aminolevulinate photodynamic therapy with cryotherapy or fluorouracil for treatment of squamous cell carcinoma in situ: results of a multicenter randomized trial. Arch Dermatol 2006; 142: 729-735.

13. Salim A, Leman JA, McColl JH et al. Randomized comparison of photodynamic therapy with topical 5-fluorouracil in Bowen's disease. Br J Dermatol 2003; 148: 539-543.

14. Patel GK, Goodwin R, Chawla M et al. Imiquimod $5 \%$ cream monotherapy for cutaneous squamous cell carcinoma in situ (Bowen's disease): a randomized, double-blind, placebo-controlled trial. J Am Acad Dermatol 2006; 54: 1025-1032.

15. Peris $\mathrm{K}$, Micantonio T, Fargnoli MC et al. Imiquimod 5\% cream in the treatment of Bowen's disease and invasive squamous cell carcinoma. J Am Acad Dermatol 2006; 55: 324-327.

16. Mackenzie-Wood A, Kossard S, de Launey J et al. Imiquimod $5 \%$ cream in the treatment of Bowen's disease. J Am Acad Dermatol 2001; 44: 462-470.

17. Cox NH, Dyson P. Wound healing on the lower leg after radiotherapy or cryotherapy of Bowen's disease and other malignant skin lesions. Br J Dermatol 1995; 133: 60-65.
18. Ashby MA, Pacella JA, de Groot R et al. Use of radon mould technique for skin cancer: Results from the Peter MacCallum Cancer Institute (1975-1984). Br J Radiol 1989; 62: 608-612.

19. Van Gestel KM, Buurman DJ, Pijls R et al. Locally advanced verrucous carcinoma of the oral cavity: treatment using customized mold HDR brachytherapy instead of hemi-maxillectomy. Strahlenther Onkol 2013; 189: 894-898.

20. Liebmann A, Pohlmann S, Heinicke F et al. Helmet moldbased surface brachytherapy for homogeneous scalp treatment: a case report. Strahlenther Onkol 2007; 183: 211-214.

21. Gauden R, Pracy M, Avery AM et al. HDR brachytherapy for superficial non-melanoma skin cancers. J Med Imaging Radiat Oncol 2013; 57: 212-217.

22. Tormo A, Celada F, Rodriguez $S$ et al. Non-melanoma skin cancer treated with HDR Valencia applicator: clinical outcomes. J Contemp Brachytherapy 2014; 6: 167-172.

23. Kowalik L, Lyczek J, Sawicki M et al. Individual applicator for brachytherapy for various sites of superficial lesions. J Contemp Brachytherapy 2013; 5: 45-49.

24. Guix B, Finestres F, Tello J et al. Treatment of skin carcinomas of the face by high-dose-rate brachytherapy and custom-made surface molds. Int J Radiat Oncol Biol Phys 2000; 47: 95-102.

25. Chung YL, Lee JD, Bang D et al. Treatment of Bowen's disease with a specially designed radioactive skin patch. Eur J Nucl Med 2000; 27: 842-846. 